\title{
Potential Regulators Driving the Transition in Nonalcoholic Fatty Liver Disease: a Stage-Based View
}

\author{
Yi Lou ${ }^{a, b} \quad$ Yi-Dan Chen ${ }^{a} \quad$ Fu-Rong Sun ${ }^{a, c} \quad$ Yu Song ${ }^{a} \quad$ Jun-Ping Shi ${ }^{a} \quad$ Jin Yang ${ }^{a}$ \\ aCenter for Translational Medicine, The Affiliated Hospital of Hangzhou Normal University, Hangzhou, \\ Zhejiang, 'Department of occupational medicine, Hangzhou Red Cross Hospital, Hangzhou, Zhejiang, \\ 'Department of Elderly Gastroenterology, The First Affiliated Hospital of China Medical University, \\ Shenyang, Liaoning, China
}

\section{Key Words}

NAFLD • Transcription factor • Network

\begin{abstract}
Background and Aim: The incidence of nonalcoholic fatty liver disease (NAFLD), ranging from mild steatosis to hepatocellular injury and inflammation, increases with the rise of obesity. However, the implications of transcription factors network in progressive NAFLD remain to be determined. Methods: A co-regulatory network approach by combining gene expression and transcription influence was utilized to dissect transcriptional regulators in different NAFLD stages. In vivo, mice models of NAFLD were used to investigate whether dysregulated expression be undertaken by transcriptional regulators. Results: Through constructing a large-scale coregulatory network, sample-specific regulator activity was estimated. The combinations of active regulators that drive the progression of NAFLD were identified. Next, top regulators in each stage of NAFLD were determined, and the results were validated using the different experiments and bariatric surgical samples. In particular, Adipocyte enhancer-binding protein 1 (AEBP1) showed increased transcription activity in nonalcoholic steatohepatitis (NASH). Further characterization of the AEBP1 related transcription program defined its co-regulators, targeted genes, and functional organization. The dynamics of AEBP1 and its potential targets were verified in an animal model of NAFLD. Conclusions: This study identifies putative functions for several transcription factors in the pathogenesis of NAFLD and may thus point to potential targets for therapeutic interventions.
\end{abstract}

\section{Introduction}

Non alcoholic fatty liver disease (NAFLD), defined as the presence of lipid accumulation in the liver ( $>5 \%$ of hepatocytes), has become the main cause of liver disease today, with worldwide prevalence in the general population estimated to be $20-30 \%$ in Western countries and $5-18 \%$ in Asia [1]. While pure steatosis (nonalcoholic fatty liver, NAFL) is a largely

Jun-Ping Shi

Jin Yang
Center for Translational Medicine, The Affiliated Hospital of Hangzhou Normal

University, Hangzhou, Zhejiang (China)

E-Mail hz_zhiy@163.com / davidshi0571@126.com

\section{KARGER}




\section{Cellular Physiology Cell Physiol Biochem 2017;41:239-251 \begin{tabular}{ll|l} 
DOI: 10.1159/000456061 & ( ) 2017 The Author(s). Published by S. Karger AG, Basel
\end{tabular}

Lou et al.: Transcriptional Co-Regulatory Network of NAFLD

benign condition, it can be complicated by the development of nonalcoholic steatohepatitis (NASH), which can progress to cirrhosis, liver failure or hepatocellular carcinoma (HCC). The precise etiology of NAFLD is unknown but there is a strong association with obesity, metabolic syndrome and dyslipidemia, among other factors.

Correspondingly, the pathogenesis of NAFLD is multifactorial. Currently, oxidative stress, inflammation, insulin resistance, nutritional effectors, lipogenesis, gut microbiota, and genetic factors are all recognized as important contributors to NAFLD [2, 3]. However, knowledge of the pathogenesis of NAFLD progression, especially the transition mode in each stage of NAFLD is still incomplete.

Transcriptomics is now the most commonly available type of genomic data, and it reflects the functional states of a tissue and determines the dominant phenotype of cells. For this determination, reconstruction of gene regulatory networks (GRNs) is an important step for disclosing the complex regulatory mechanisms that are involved in forming the cell phenotype. The prominent transcription regulators, transcription factors (TFs), can act as activators or repressors simultaneously in a cellular context. Moreover, the complexity of regulation and expression of targeted genes is often determined by the coordinated action of TFs [4]. Identifying a master regulator and its cooperative factor remains a major challenge in NAFLD research.

Herein, we present the first step towards the construction of a mechanistic model underlying NAFLD progression by the identification of the sets of TFs that potentially maintain a corresponding phenotype. We first applied GRN inference to publicly available microarray data covering the natural history of NAFLD. Active transcription factor regulators were then identified using a computational approach CoRegNet [5]. Finally, functional annotations provided evidence for the dominant roles of master regulators in NASH.

\section{Materials and Methods}

\section{Data acquisition and description}

Table 1 describes the NAFLD datasets that were used in this study. The table reports the sample size, tissue source, mean age, and the identifier of the dataset in the Gene Expression Omnibus (GEO) or ArrayExpress database.

All samples had no evidence of viral hepatitis, hemochromatosis, or alcohol over-consumption and were clinically characterized as previously described [6]. Full experimental methods and detailed descriptions of these public datasets can be found in the original references. Of note, bariatric surgery samples for NAFLD could only be found in the GSE48452 dataset [7]. The surgical samples were mostly from obese patients (before/after surgery: 4/11) or NAFL patients (before/after surgery: 7/5). These samples were extracted and used in validation analysis.

\section{Transcriptional co-regulatory network}

Transcriptome analysis was performed using the R software and Bioconductor Affy package [8]. Raw expression data were normalized by a robust multi-array average (RMA) function. After the removal of genes that were low- or un-expressed, the top 3000 varying genes were considered for further analysis. Differential expression was detected using the limma package and the significance threshold was the false discovery rate (FDR) $<0.01[9]$.

To identify the NAFLD-driving active regulatory circuits, a Bioconductor package CoRegNet was used. Briefly, the cooperative regulation networks were inferred using the h-Licorn algorithm, which is able to identify experimentally validated regulator cooperative relationships solely based on gene expression data [10]. To obtain a more confident network, input features such as the TF-gene interaction (e.g., chromatin modification datasets and TF binding site) [11] and TF-TF interaction data (e.g., protein interactions [12]) were integrated to support cooperative interactions. The inferred network was refined by selecting a subset of the network that is enriched in this external regulatory and co-regulatory evidence using an integrative classifier algorithm [13]. 


\section{Cellular Physiology Cell Physiol Biochem 2017;41:239-251

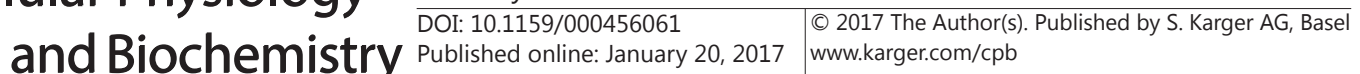

Lou et al.: Transcriptional Co-Regulatory Network of NAFLD

Table 1. Description of gene expression data sets. *Note that the gender differs greatly across the studies. There were totally 19 cases receiving bariatric surgeries in GSE48452 dataset. ${ }^{\$}$ NA: Not Applicable

\begin{tabular}{|c|c|c|c|c|c|c|c|c|}
\hline Set & $\mathrm{n}$ & Tissue & Description & Gender(female:male) ${ }^{*}$ & $\begin{array}{c}\text { Age } \\
(m e a n \pm s d)\end{array}$ & NAS(mean \pm sd) & Platform & $\begin{array}{l}\text { Public } \\
\text { availability }\end{array}$ \\
\hline \multirow[t]{4}{*}{1} & 73 & liver & 14 normal & 9:5 (including 2 post-surgery samples) & $51.83 \pm 17.68$ & $0.07 \pm 0.27$ & Affymetrix & GSE48452* \\
\hline & & & 27 obesity & 25:2(including 11 post-surgery samples) & $45.35 \pm 7.30$ & $0.15 \pm 0.46$ & HuGene 1.1 & \\
\hline & & & 14steatotic & 10:4 (including 5 post-surgery samples) & $41.60 \pm 11.22$ & $1.71 \pm 0.83$ & ST gene & \\
\hline & & & $18 \mathrm{NASH}$ & 14:4 (including 1 post-surgery samples) & $45.48 \pm 8.93$ & $5.06 \pm 0.87$ & & \\
\hline \multirow[t]{3}{*}{2} & 45 & liver & 19 normal & $9: 10$ & $43.26 \pm 19.19$ & $\mathrm{NA}^{\mathrm{s}}$ & Affymetrix & E-MEXP- \\
\hline & & & 10 steatotic & $5: 4$ (one unknown sex) & $44.67 \pm 14.95$ & NA & HuGene 1.0 & 3291 \\
\hline & & & $16 \mathrm{NASH}$ & $12: 3$ (one unknown sex) & $55.93 \pm 7.94$ & NA & ST gene & \\
\hline
\end{tabular}

\section{Transcriptional influence and network visualization}

Transcription factor influence was used to estimate the activity of a TF in a given sample. Based on a comparison of the expression of the activated and the repressed targets of a regulator, the influence was computed in a sample-specific manner [5]. For integrative visualization, the network was accessible through a Cytoscape [14] widget with functionalities to display sets of active co-regulators in particular samples.

\section{Gene Ontology Analysis}

GO analyses for biological process and KEGG pathway enrichment were performed using the Database for Annotation, Visualization and Integrated Discovery (DAVID) [15]. In DAVID, an overrepresentation of a term or pathway is defined as a modified Fisher's exact P-value with an adjustment for multiple tests using the Benjamani-Hochberg method. In addition, we related TFs to biological relevance in NAFLD on the basis of the literature data.

\section{Experimental animals and diets}

ApoE $/$-mice on a C57BL/6J background were purchased (Model Animal Research Center of Nanjing University, China) at 4 weeks of age. ApoE $/$ mice were divided into two groups and allocated into either a normal Chow-diet (Normal group) or high fat high cholesterol-diet (HFHC group).

The HFHC diet was from Research Diets, New Brunswick (D12079B, Research Diets, New Brunswick, NJ), which contained $21 \%$ fat, $20 \%$ protein, $50 \%$ starch and $0.21 \%$ cholesterol. At the end of the experiments, a part of the liver tissue was fixed with $10 \%$ formaldehyde, and the remaining liver was snap frozen. All animals received humane care, and all protocols dealing with animals were reviewed and approved by the local animal ethics committee.

\section{Liver histology}

Formalin-fixed liver tissue was processed into $4 \mu \mathrm{m}$ thick paraffin sections and stained with hematoxylin and eosin as previously described [16]. The degree of steatosis (0-3), lobular inflammation (0-3), hepatocyte ballooning (0-2), and NAFLD activity score (NAS) were scored according to NASH clinical research network (CRN) scoring system.

\section{Total RNA extraction and quantitative RT-PCR analysis}

Hepatic mRNA levels were analyzed by qRT-PCR using a 7900 Real Time PCR System (Applied Biosystems, USA). The total RNA of the liver tissues was isolated with TRIzol (Invitrogen, USA). cDNA was synthesized using $2 \mu \mathrm{g}$ of total RNA with PrimeScript ${ }^{\mathrm{TM}}$ Reverse Transcriptase (Takara). Amplification reactions were performed using the SYBR $®$ Premix Ex Taq kit (Takara) and $0.2 \mu \mathrm{M}$ of gene specific primers (for all online suppl. material, see www.karger.com/doi/000456061 Table S1) and PCR products were verified by melting curve analysis. The relative quantification expression was calculated using the deltadelta Ct method with each gene normalized to Gapdh.

\section{Results}

A landscape depicting the liver network

We first reconstructed a putative co-regulatory network from gene expression data (GSE48452) and integrated supporting regulatory evidence such as the TF binding site, 


\section{Cellular Physiology Cell Physiol Biochem 2017;41:239-251 \begin{tabular}{l|l|l|l} 
DOI: 10.1159/000456061 & (O) 2017 The Author(s). Published by S. Karger AG, Basel
\end{tabular} \\ Lou et al.: Transcriptional Co-Regulatory Network of NAFLD}

Table 2. Top 10 co-regulators (regulator pairs) across all stages * The complete co-regulator pairs based on refined GRN were provided in see online suppl. material, Table S3.The co-regulators were calculated by coregulators function in CoRegNet package and sorted by adjusted $P$ value. aFisher test determines the specificity of the shared targets between two regulators. The default p-value adjustment uses FDR correction to extract significant pairs of co-regulators

\begin{tabular}{llll}
\hline Regulator1 & Regulator2 & fisherTest $^{\mathrm{a}}$ & adjusted P-value \\
\hline EGR1 & FOS & $1.36 \mathrm{E}-119$ & $9.00 \mathrm{E}-117$ \\
JUNB & ZFP36 & $6.56 \mathrm{E}-60$ & $2.17 \mathrm{E}-57$ \\
FOS & ZFP36 & $9.41 \mathrm{E}-38$ & $2.08 \mathrm{E}-35$ \\
JUN & MYC & $2.08 \mathrm{E}-33$ & $3.45 \mathrm{E}-31$ \\
EGR1 & ZFP36 & $8.99 \mathrm{E}-30$ & $1.19 \mathrm{E}-27$ \\
FOSB & ZFP36 & $1.59 \mathrm{E}-28$ & $1.75 \mathrm{E}-26$ \\
JUNB & NFIL3 & $1.28 \mathrm{E}-27$ & $1.21 \mathrm{E}-25$ \\
NFIL3 & ZFP36 & $1.72 \mathrm{E}-26$ & $1.42 \mathrm{E}-24$ \\
CEBPA & MLXIPL & $1.12 \mathrm{E}-25$ & $8.21 \mathrm{E}-24$ \\
NR4A1 & ZFP36 & $1.78 \mathrm{E}-25$ & $1.18 \mathrm{E}-23$ \\
\hline
\end{tabular}

ChIP-seq data, and protein interactions embedded in CoRegNet [5]. In GSE48452 dataset [7], 54 cases with no bariatric surgery, were classified clinically [17] into normal controls $(n=12)$, morbid obesity $(n=16)$, NAFL $(n=9)$, and NASH $(n=17)$ groups. This dataset represents the natural history of NAFLD occurrence and progression. To reduce the heavy computation load, we used the top 3000 informative genes in GSE48452.

The activity of each TF in any given sample, and the sets of cooperative TFs were estimated (Table 2). Fig. 1 showed the representative TFs across all states, where FOS, JUN, EGR, and MYC have a high influential significance in liver physiology.

These factors trigger the early response gene expression. For instance, following the growth signal delivered by partial hepatectomy, these genes are all involved in the progression of hepatocytes through the G1 phase, leading the transition from quiescence to proliferation [18]. The EGR family of transcription factors has been implicated in the control of lipid biosynthetic genes. EGR1 is induced by insulin and is highly expressed in the liver [19]. Moreover, all these genes moderately fluctuate during all the stages of NAFLD (Fig. 1-2).

Natural history of NAFLD from a stage-based view

Next, we examined the representative regulators in each stage for this dataset. Top regulators in each stage are shown in Table 3.

In the normal state, NR0B2(also known as SHP) was the essential regulator. NR0B2 is a hepatic tumor suppressor and critical for liver function [20]. NR0B2 represses multiple genes involved in hepatic lipid metabolism and inflammation [21] and has been shown to be implicated in cholestatic liver injury due to bile acid feeding, fatty liver, liver fibrosis, and liver cancer $[22,23]$. In addition, NR0B2 is a newly identified regulator of hepatocyte apoptosis and DNA methylation [24]. NR0B2 levels are downregulated in human cirrhosis and HCC [22]. Another regulator, KLF15, is an important molecular link between ER stress and insulin action [25]. KLF15 regulates the expression of genes for gluconeogenic or amino acid-degrading enzymes [26]. Moreover, the KLF15-FGF15 signaling axis regulates circadian bile acids production [27].

In the obese state, early response transcription factors such as FOS, FOSB, and JUN were activated. Another top regulator, NR4A2, has emerged as an important nuclear receptor in the regulation of metabolic tissues, particularly in the liver [28]. NR4A2 regulates both glucose and fatty acid metabolism, thus linking NR4A2 to energy metabolism and cell survival. Its expression was significantly lower in fibrotic liver tissues [29].

In the NAFL state, MLXIPL (also named as CHREBP) was found to be the most active TF. MLXIPL has crucial roles for coordinately regulating lipogenic genes at the transcriptional level, and converts excess carbohydrate to fat storage in the liver [30]. KLF15 is reactivated in the NAFL state, which has important implications for the physiologic control of nutrient availability and metabolic homeostasis [27].

In the NASH state, AEBP1, IFI16, and PRDM1,etc were the key regulators. AEBP1 has been shown to impede macrophage cholesterol efflux, promoting foam cell formation. 


\section{Cellular Physiology Cell Physiol Biochem 2017;41:239-251

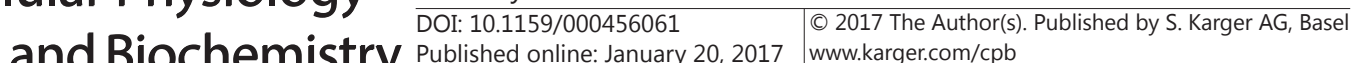

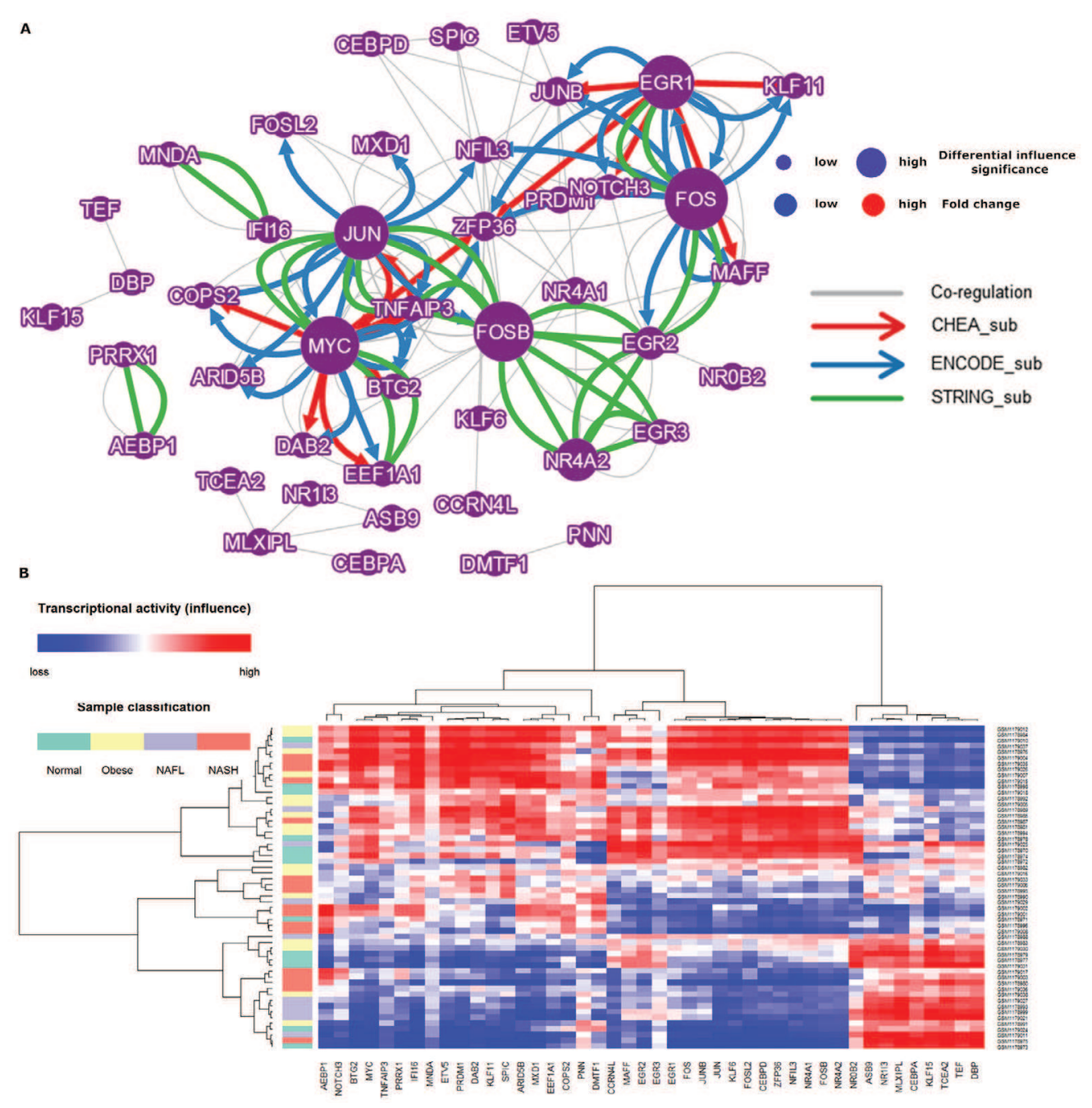

Fig. 1. Transcription co-regulators network. (A) Putative co-regulation network across all the stages in NAFLD. Cooperative TFs network from GSE48452 transcriptomic data was constructed using the h-Licorn algorithm and integrated with multiple type of interactions between co-regulators inferred by CoRegNet. A Cytoscape application is to visualize the network. (B) A heatmap shows the influence of all TFs in all samples. The figures were generated by CoRegNet package.

Moreover, AEBP1 promotes macrophage inflammatory responsiveness by inducing nuclear factor (NF)- $\kappa B$ activity [31]. IFI16 has roles in anti-proliferation, autophagy, cell senescence, anti-inflammation, and DNA sensor to trigger innate immunity. IFI16 is physiologically absent in adult healthy hepatocytes, but exists in liver cancer cells [32].

\section{Transcription program shared between datasets}

To determine if active TFs were common in different datasets, an independent validation was performed. We downloaded the gene profiling data with accession number E-MEXP-3291 to serve as the control for this dataset, which included well recorded normal, NAFL and NASH patients [6]. For ease of comparison, we first generated the top 3000 varying genes in GSE48452 and E-MEXP-3291. After intersecting the two gene lists, 1789 shared genes were used to infer the network.

In brief, we focused on the TF networks in the NAFL or NASH conditions. As expected, most regulators are well-preserved between the two datasets (see online suppl. material, 


\section{Cellular Physiology Cell Physiol Biochem 2017;41:239-251

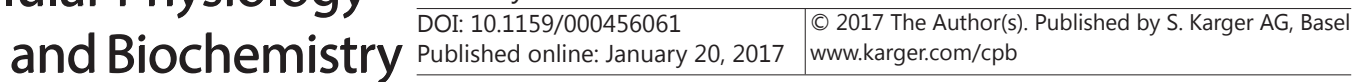

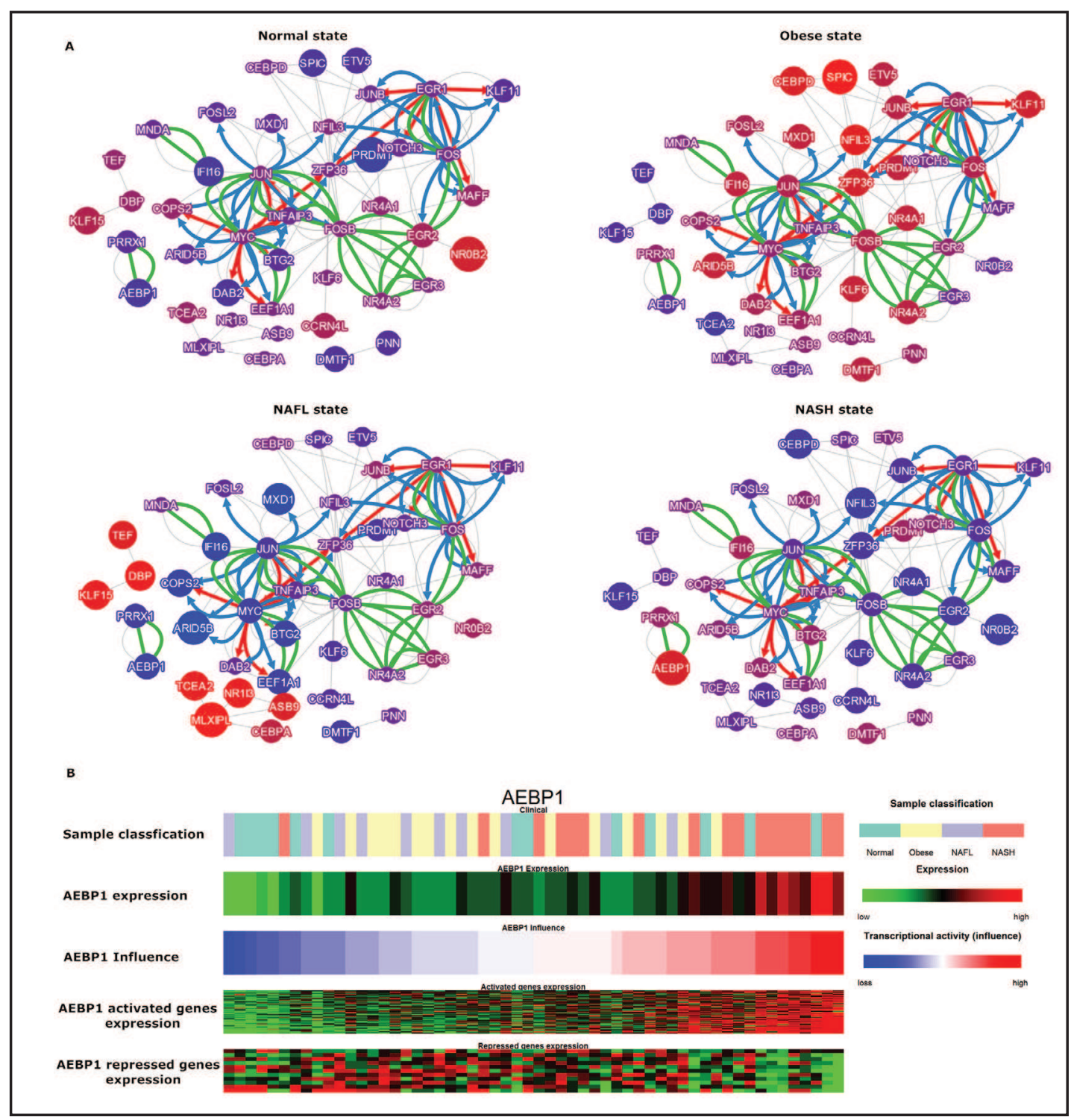

Fig. 2. Transcription co-regulators network in each stage of NAFLD. (A) TFs co-regulation in the normal, obese, NAFL and NASH state respectively. The meaning of nodes and links follow the legends as illustrated in Fig. 1. (B) AEBP1 is the most representative TF in the NASH state. AEBP1 related heatmaps are showed in the lower panel. Heatmaps display one sample per column. The first heatmap color codes the sample classification. The second and third show the expression and influence values of AEBP1. The fourth and fifth heatmap display the expression of the activated and repressed genes respectively.

Fig. S1). In the NAFL state, regulators such as MLXIPL, KLF15 and SPIC, etc. were activated. In the NASH state, AEBP1, IFI16, and PRDM1, etc., showed significant transcriptional activity.

Because bariatric surgery is the most radical treatment for obesity and NAFLD, and as described earlier, bariatric patients showed expected improvement in liver histology [7], we next performed the GRN using the before- and after- surgery samples in GSE42825 to observe if the representative regulators were remodeled after bariatric surgery. Of note, most surgery cases in GSE48452 belong to obese or NAFL patients (obese, before/after surgery: 4/11; NAFL, before/after surgery: 7/5). As expected, TFs such as NR4A2, FOSB, SPIC, MLXIPC, etc., were enriched before surgery. All these potential driver regulators in obese or NAFL patients were inversely changed after surgery, respectively (see online suppl. material, Fig. S2). 
Table 3. Master regulator in each NAFLD stage. * Transcription influence (Mean \pm SEM)

\begin{tabular}{lcllllll}
\hline \multicolumn{2}{c}{ Normal } & \multicolumn{2}{c}{ Obesity } & \multicolumn{2}{c}{ NAFL } & \multicolumn{2}{c}{ NASH } \\
\hline NR0B2 & $3.56 \pm 0.33^{*}$ & FOSB & $3.77 \pm 0.41$ & MLXIPL & $5.85 \pm 0.75$ & AEBP1 & $3.23 \pm 0.32$ \\
KLF15 & $2.41 \pm 0.58$ & NR4A2 & $3.44 \pm 0.26$ & KLF15 & $4.38 \pm 0.71$ & IFI16 & $3.21 \pm 0.78$ \\
EGR2 & $1.97 \pm 0.44$ & SPIC & $3.09 \pm 0.26$ & TCEA2 & $3.64 \pm 0.55$ & PRDM1 & $2.53 \pm 0.84$ \\
\hline
\end{tabular}

Table 4. Functional annotation of AEBP1 transcription program

\begin{tabular}{llll}
\hline AEBP1 activation program & G0TERM_BP_DIRECT Term & PValue & FDR \\
\cline { 2 - 3 } & G0:0030198 extracellular matrix organization & $4.14 \mathrm{E}-17$ & $6.45 \mathrm{E}-14$ \\
& G0:0022617 extracellular matrix disassembly & $1.61 \mathrm{E}-09$ & $2.51 \mathrm{E}-06$ \\
& G0:0030199 collagen fibril organization & $9.85 \mathrm{E}-08$ & $1.53 \mathrm{E}-04$ \\
& G0:0007155 cell adhesion & $7.11 \mathrm{E}-07$ & $1.11 \mathrm{E}-03$ \\
& G0:0001525 angiogenesis & $4.70 \mathrm{E}-06$ & $7.32 \mathrm{E}-03$ \\
& KEGG_PATHWAY & & \\
& hsa04510:Focal adhesion & $1.36 \mathrm{E}-06$ & $1.61 \mathrm{E}-03$ \\
& hsa04512:ECM-receptor interaction & $3.08 \mathrm{E}-05$ & $3.66 \mathrm{E}-02$ \\
& hsa05205:Proteoglycans in cancer & $2.61 \mathrm{E}-03$ & $3.05 \mathrm{E}+00$ \\
& hsa04151:PI3K-Akt signaling pathway & $2.71 \mathrm{E}-03$ & $3.17 \mathrm{E}+00$ \\
& hsa04670:Leukocyte transendothelial migration & $9.57 \mathrm{E}-03$ & $1.08 \mathrm{E}+01$ \\
& & & \\
& G0TERM_BP_DIRECT Term & PValue & FDR \\
& G0:0042102 positive regulation of T cell proliferation & $3.38 \mathrm{E}-03$ & $4.07 \mathrm{E}+00$ \\
& G0:0045087 innate immune response & $9.63 \mathrm{E}-03$ & $1.12 \mathrm{E}+01$ \\
& G0:0006955 immune response & $1.52 \mathrm{E}-02$ & $1.71 \mathrm{E}+01$ \\
& G0:0002250 adaptive immune response & $1.96 \mathrm{E}-02$ & $2.16 \mathrm{E}+01$ \\
& G0:0006953 acute-phase response & $6.07 \mathrm{E}-02$ & $5.36 \mathrm{E}+01$ \\
& KEGG_PATHWAY repression program & & \\
& hsa04610:Complement and coagulation cascades & $1.51 \mathrm{E}-02$ & $1.36 \mathrm{E}+01$ \\
hsa04514:Cell adhesion molecules (CAMs) & $5.74 \mathrm{E}-02$ & $4.32 \mathrm{E}+01$ \\
\hline
\end{tabular}

AEBP1 transcription program

Because AEBP1 activity is highly enhanced in the NASH state in different experiments, we next explored the functional organization of AEBP1 program. In total, 102 genes were predicted to be activated by AEBP1, while 30 genes were predicted to be repressed (Fig.3). Using the DAVID annotation, the five highest ranking gene ontology (GO) analysis terms and KEGG pathways are listed in Table 4. In terms of the AEBP1 activation program, focal adhesions and extracellular matrix organization were the leading GO terms and KEGG pathways. The GO terms specific for the AEBP1 repression program revealed terms that shared $\mathrm{T}$ cell proliferation and immune response as a common theme.

To more precisely demonstrate the role of the AEBP1 program, the co-regulators of AEBP1 were computed. In complete accord with the AEBP1 program, the extracellular matrix organization was the dominant functional annotation for the pairing of AEBP1 with co-regulators. A full list, including targeted genes, GO terms and pathway annotation is provided in (see online suppl. material, Table S2).

Aebp1 dynamic in the animal model

ApoE $/-$ mice receiving a HFHC diet comprise the well-established animal model used for studies of NAFLD [16]. As expected, $\mathrm{ApoE}^{-/-}$mice fed a HFHC diet for 12-16 weeks in this study showed hepatic steatosis, ballooning and increased hepatic inflammation (Fig. 4A), which recapitulated the clinical findings in human NAFLD.

For the initial step in the observation of the AEBP1 program in NAFL (12 weeks) and NASH (16 weeks) state, we measured mRNA levels of liver Aebp1 in Normal, NAFL and NASH mice. There was no difference in the mRNA abundance of Aebp1 between Normal and NAFL mice. In contrast, Aebp1 was higher in the livers of NASH mice compared to the livers of NAFL mice.

Compared to the HFHC diet fed to the mice at 12 weeks, genes contributing to the G0:0030198-extracellular matrix organization such as Efemp1, Fabp4, Fbln5, Lum, Mmp2, $\mathrm{Tgfb} 2$ and Thbs2, were up-regulated at 16 weeks, when a more typical NASH state appeared. For genes contributing to the GO:0042102-positive regulation of $\mathrm{T}$ cell proliferation, $\mathrm{Cd} 4$ 


\section{Cellular Physiology Cell Physiol Biochem 2017;41:239-251

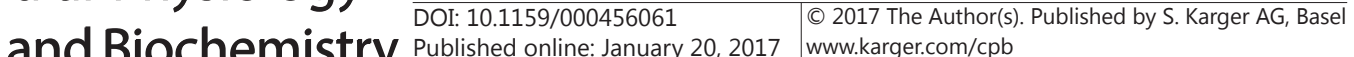

Lou et al.: Transcriptional Co-Regulatory Network of NAFLD

showed a tendency to decrease in the NASH state (Fig. 4B). These findings identified the relationship between AEBP1 and ECM target genes during NAFLD progression.

\section{Discussion}

NAFLD is a complex condition comprising multiple independent modifiers of risk, such as obesity and insulin resistance [33]. The link among obesity, liver steatosis, lipotoxicity, inflammation and cancer has raised more concern recently,which is described as "the oil that feeds the flame" [34-36].

Gene expression signatures are not static and can be reprogrammed by TFs. Our results provide several new insights into dynamic co-regulatory networks across the natural history of NAFLD.

We found that the h-Licorn algorithm and CoRegNet package used in regulatory network inference plausibly captures regulatory interactions. In this study, TFs such as FOS, JUN, and ERG constituted the main components of the network, where protein interaction evidence (STRING) and functional TF information (Encode patterns [37]) were markedly converged. Another feature of these TFs is the fluctuation among different NAFLD states. Indeed, c-Jun/activator protein 1 (AP-1) is strongly expressed in response to inflammatory stimuli, promotes hepatocyte survival during acute hepatitis and acts as an oncogene during chemically induced liver carcinogenesis. JUN has important functions during HBV-associated tumorigenesis by promoting hepatocyte proliferation as well as progression of dysplasia [38]. Moreover, previous studies have introduced the term liver-enriched transcription factors (LETFs), which point to pivotal roles of albumin D site-binding protein (DBP) and the CAAT/enhancer-binding proteins (C/EBPs) in cell cycle control, circadian gene regulation, liver regeneration, apoptosis, and liver-specific gene regulation [39, 40]. As shown in Fig. 1, DBP, CEBPA, and CEBPD were presented in the refined network.

GRN is a highly dynamic process, due in part to the heterogeneity of the genomic features. Thus, we applied a cross-validation between two well-recorded sets (GSE48452 and E-MEXP-3291) to assess the quality of inferred regulatory networks. First, given NAFL or NASH as examples, transcription patterns are reproducible for most TFs between two different experiments. Second, across the public gene expression database, bariatric surgery samples for NAFLD could only be found in GSE48452 dataset. Here, surgical samples also verified similar TF remodeling events.

Another major reason for selecting GSE48452 is that it represents the multiple phases of NAFLD; thus, it may present the global dynamic picture of NAFLD states. We provided the representative regulators in each stage of NAFLD. Apart from the factors listed in the text, lots of regulators have been documented in liver pathogenesis. For instance, NOTCH3 is the key factor in the process of fetal liver stem/progenitor cell differentiation into hepatocytes [41, 42] (Fig. 2). NOTCH3 also regulates the activation of hepatic stellate cells, and is involved in liver fibrosis [43]. PRRX1 restrains adipogenesis by regulating expression of TGF- $\beta$ ligands and thereby activates TGF- $\beta$ signaling [44]. The BTG2 gene participates in the regulation of hepatic glucose homeostasis [45]. Importantly, we provided new functional links for several previously unreported TFs for NAFLD disease. For example, the Ets transcription factor SPIC is activated in the morbidly obese state. SPIC is expressed in B cells and macrophages, and is closely related to cell differentiation [46]. SPIC controls tissue macrophages activity [47]. TCEA2(TFIIS) is activated in the NAFL state (Fig. 2). TCEA2 functions as a transcription elongation factor and is important for preventing cellular death due to oxidative DNA damage [48]. PRDM1, also known as B-lymphocyte-induced maturation protein 1 (BLIMP1), is activated in the NASH state (Fig. 2). An important role for PRDM1 in maintaining the homeostasis of effector T cells is emerging. PRDM1 mediates the development of tissueresident memory $\mathrm{T}$ cells in the skin, gut, and liver [49]. However, their roles in NAFLD progression have not yet been reported. The possibility of these factors controlling lymphocytic infiltration with the progression of disease needs further investigation. 


\section{Cellular Physiology Cell Physiol Biochem 2017;41:239-251 and Biochemistry DOI: 10.1159/000456061 20, 2017 O 2017 The Author(s). Published by S. Karger AG, Basel}

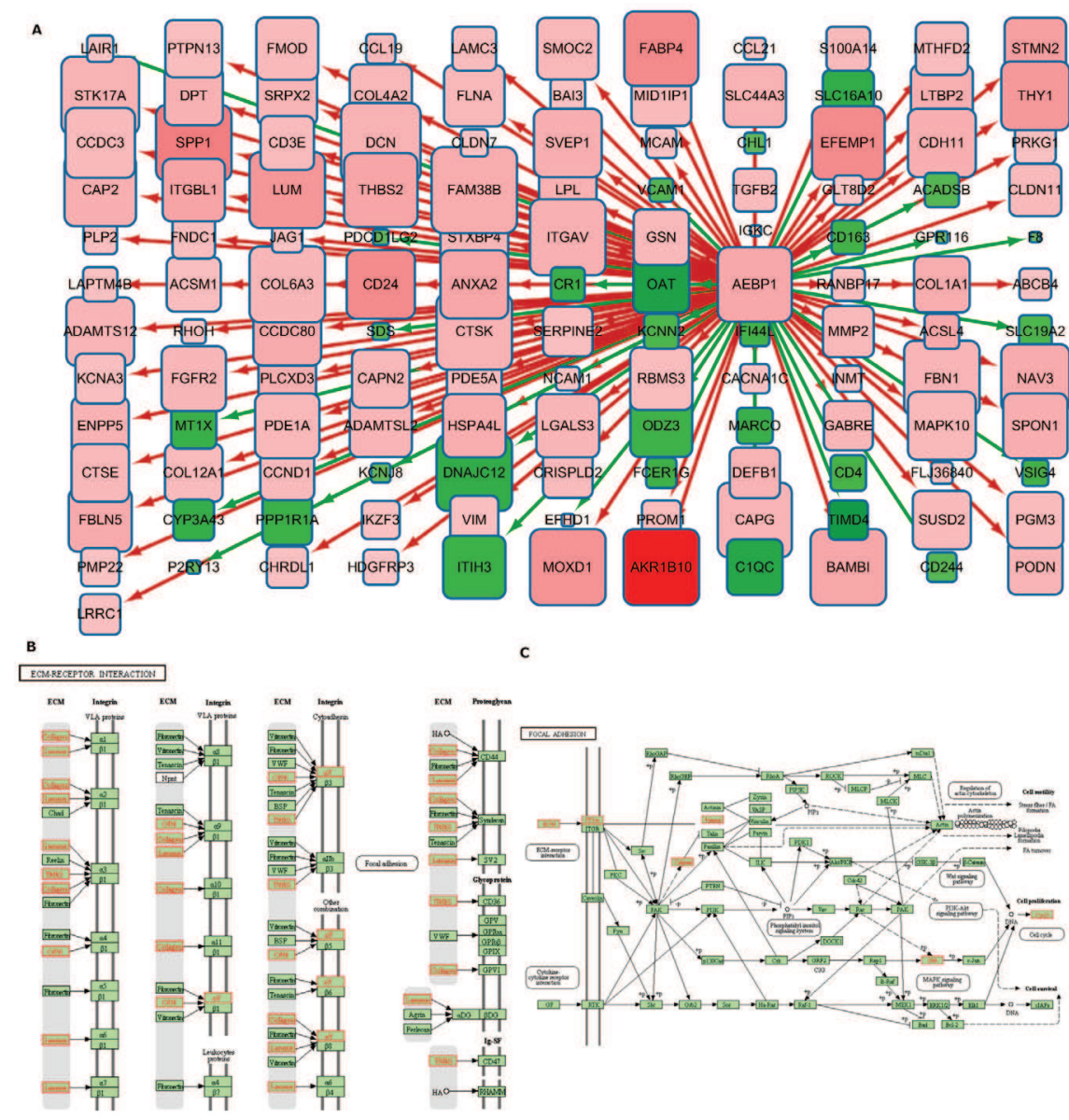

Fig. 3. AEBP1 transcription program . (A) Targeted genes of AEBP1. The node color coded from green to red (low to high) indicate the fold change when compared NASH with NAFL state. The node size is proportional to the significance of the expression changes compared to NAFL. The green and red edges indicate if AEBP1 activate or repress the targeted gene, respectively. (B) ECM pathway. (C) Focal adhesion pathway. Red boxes represent enriched genes.

Different genomic backgrounds may contribute to differences in signature, thus some unique TFs were presented in E-MEXP-3291. For instance, in the NAFL state, CREB3L3 is the most activated transcription factor. Indeed, CREB3L3 is highly and selectively expressed in the liver, and activated by ER stress or inflammatory stimuli to induce an acute-phase hepatic inflammation. CREB3L3 is a key metabolic regulator of hepatic lipogenesis, fatty acid oxidation, and lipolysis under metabolic stress, thus controlling whole-body energy homeostasis [50]. In the NASH state, the relevance of PPARGC1A to NAFLD has been well documented; a tight interaction of the insulin resistance phenotype to the differential susceptibility of mice to hepatic steatosis was shown $[7,51]$. One of its co-regulators, NR4A1 regulates TGF- $\beta$ signaling and fibrosis [52]. All of these examples suggest that our predicted cooperative TFs are promising and interesting subjects for future experiments.

Since steatosis is generally a stable disease whereas NASH can be more progressive, we placed emphasis on the transition from NAFL to NASH. AEBP1 is the key TF during this process. Using traditional differential analysis, we showed that no significant difference was observed when only considering AEBP1 expression $(6.42 \pm 0.33$ vs. $7.16 \pm 0.66$, in the NAFL vs. NASH state, respectively; q value $=0.069$; fold change $=1.66$; $B=-1.409$ ). However, AEBP1 confers marked transcriptional activity in NASH. Indeed, previous reports have not linked AEBP1 to the NAFLD phenotype. In this study, directed targeted genes including activation 


\section{Cellular Physiology \\ Cell Physiol Biochem 2017;41:239-251 and Biochemistry

Fig. 4. Dynamics of Aebp1-related genes under different NAFLD state. (A) As compared to mice fed with Chow diet, mice fed with HFHC diet for 12 16 weeks exhibited pathological findings of NAFLD. Representative slides show hematoxylin and eosin (H\&E)-stained liver sections from NAFL mice (12 weeks, NAFLD activity score $=3$ ) or NASH mice (16 weeks, NAS=6). (B) mRNA levels of Efemp1, Fabp4, Fbln5, Lum, Mmp2, Tgfb2, Thbs2 and Cd4 in the NAFL and NASH state, respectively. Relative mRNA levels were determined by real-time RT-PCR and were normalized to Gapdh mRNA levels under normal state. Data are described as the means \pm SEM of $(n=3 \sim 5$ mice per group).

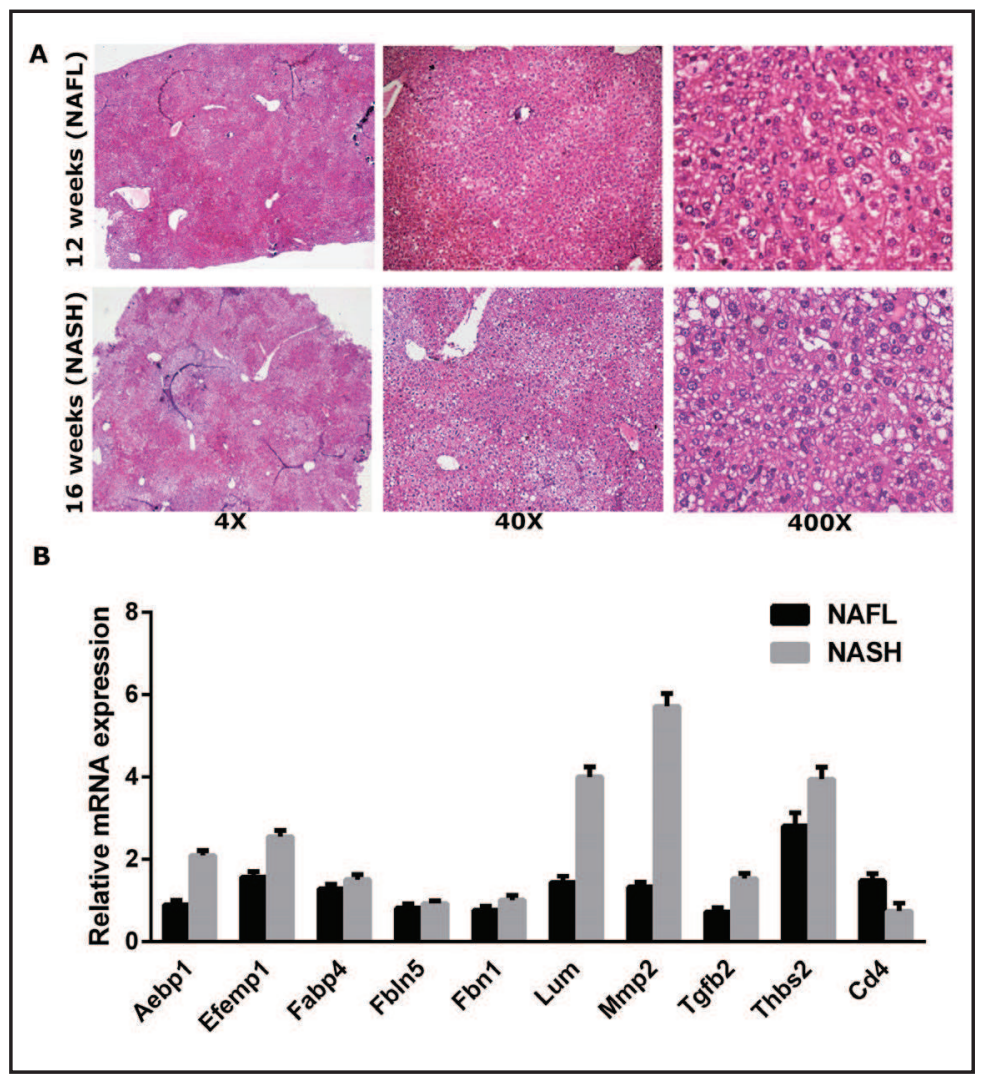

and repression effects of AEBP1 were described. The functional organization of the AEBP1 program focuses on the role of promoting ECM and repressing the immune response. This notion was strengthened by reviewing the AEBP1-coregulator pairs in the network, where highly concordant GO terms and pathways were enriched (see online suppl. material, Table S2), thereby implicating the importance of AEBP1 in NAFLD. It is worth noting that the GSE48452 dataset did not include patients with advanced NASH and high grades of fibrosis. Similarly, in E-MEXP-3291, no fibrosis scores were provided for NASH patients. Again, AEBP1-coregulators as well as the functional annotation were highly reproduced between the two datasets. Moreover, we used $\mathrm{ApoE}^{-/-}$mice receiving a HFHC diet as a typical model of obesity-induced NASH [53]. We selected the potential targets from the top GoTerm in the AEBP1 program, and verified that AEBP1 activation in the NASH state is associated with the genes that are up-regulated in ECM remodeling (Fig. 3-4). Therefore, it is highly probable that AEBP1 is the regulator that initiates fibrosis, which is the strongest predictor for diseasespecific mortality in NAFLD [54].

Currently, several human NAFLD transcriptome studies have been reported. One group used weighted gene co-expression network analysis to dissect transcriptional profiles and found 9 modules of co-expressed genes associated with NAFL/NASH transition [55]. Several groups integrated transcriptomics and lipidomics to exploit the metabolic adaptability during disease progression $[56,57]$. To our knowledge, no transcription factor networks have ever been performed. Several lines of evidence suggest that the networks constructed here are biologically significant. When validated, these candidate TFs can provide molecular targets for diagnosis and therapy.

In conclusion, our work provides an initial step toward identifying cooperative TFs in the natural history of NAFLD. We provided unique insights into the different regulatory mechanisms associated with NAFLD. Specifically, we successfully identified the stable AEBP1 transcription program in the NASH state. In addition, we identified many novel and potentially cooperative TFs that could lead directly to new hypotheses for future experiments. 


\section{Cellular Physiology Cell Physiol Biochem 2017;41:239-251

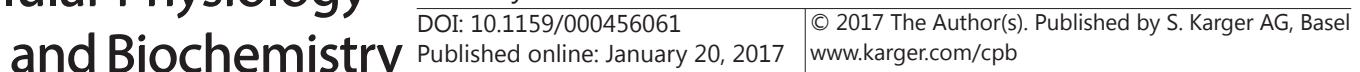

Lou et al.: Transcriptional Co-Regulatory Network of NAFLD

\section{Funding}

Thiswork wassupported bythe National Natural Science Foundation of China(81570524), Zhejiang Provincial Natural Science Foundation(LY15H070004, LY14H190001), and Major project of Hangzhou health and family planning science and technology project (2015ZD02). The funders had no role in study design, data collection and analysis, decision to publish, or preparation of the manuscript.

\section{Disclosure Statement}

The authors declare that they have no conflicts of interest.

\section{Reference}

1 Masarone M, Federico A, Abenavoli L, Loguercio C, Persico M: Non alcoholic fatty liver: Epidemiology and natural history. Rev Recent Clin Trials 2014;9:126-133.

-2 Feng YY, Xu XQ Ji CB, Shi CM, Guo XR, Fu JF: Aberrant hepatic microrna expression in nonalcoholic fatty liver disease. Cell Physiol Biochem 2014;34:1983-1997.

-3 Bitter A, Nussler AK, Thasler WE, Klein K, Zanger UM, Schwab M, Burk O: Human sterol regulatory elementbinding protein 1a contributes significantly to hepatic lipogenic gene expression. Cell Physiol Biochem 2015;35:803-815.

-4 Neph S, Vierstra J, Stergachis AB, Reynolds AP, Haugen E, Vernot B, Thurman RE, John S, Sandstrom R, Johnson AK, Maurano MT, Humbert R, Rynes E, Wang H, Vong S, Lee K, Bates D, Diegel M, Roach V, Dunn D, Neri J, Schafer A, Hansen RS, Kutyavin T, Giste E, Weaver M, Canfield T, Sabo P, Zhang M, Balasundaram G, Byron R, MacCoss MJ, Akey JM, Bender MA, Groudine M, Kaul R, Stamatoyannopoulos JA: An expansive human regulatory lexicon encoded in transcription factor footprints. Nature 2012;489:83-90.

5 Nicolle R, Radvanyi F, Elati M: Coregnet: Reconstruction and integrated analysis of co-regulatory networks. Bioinformatics 2015;31:3066-3068.

6 Lake AD, Novak P, Fisher CD, Jackson JP, Hardwick RN, Billheimer DD, Klimecki WT, Cherrington NJ: Analysis of global and absorption, distribution, metabolism, and elimination gene expression in the progressive stages of human nonalcoholic fatty liver disease. Drug Metab Dispos 2011;39:1954-1960.

7 Ahrens M, Ammerpohl O, von Schonfels W, Kolarova J, Bens S, Itzel T, Teufel A, Herrmann A, Brosch M, Hinrichsen H, Erhart W, Egberts J, Sipos B, Schreiber S, Hasler R, Stickel F, Becker T, Krawczak M, Rocken C, Siebert R, Schafmayer C, Hampe J: DNA methylation analysis in nonalcoholic fatty liver disease suggests distinct disease-specific and remodeling signatures after bariatric surgery. Cell Metab 2013;18:296-302.

8 Gautier L, Cope L, Bolstad BM, Irizarry RA: Affy--analysis of affymetrix genechip data at the probe level. Bioinformatics 2004;20:307-315.

-9 Ritchie ME, Phipson B, Wu D, Hu Y, Law CW, Shi W, Smyth GK: Limma powers differential expression analyses for rna-sequencing and microarray studies. Nucleic Acids Res 2015;43:e47.

10 Chebil I, Nicolle R, Santini G, Rouveirol C, Elati M: Hybrid method inference for the construction of cooperative regulatory network in human. IEEE Trans Nanobioscience 2014;13:97-103.

11 Consortium EP: A user's guide to the encyclopedia of DNA elements (encode). PLoS Biol 2011;9:e1001046.

12 Szklarczyk D, Franceschini A, Wyder S, Forslund K, Heller D, Huerta-Cepas J, Simonovic M, Roth A, Santos A, Tsafou KP, Kuhn M, Bork P, Jensen LJ, von Mering C: String v10: Protein-protein interaction networks, integrated over the tree of life. Nucleic Acids Res 2015;43:D447-452.

13 Marbach D, Roy S, Ay F, Meyer PE, Candeias R, Kahveci T, Bristow CA, Kellis M: Predictive regulatory models in drosophila melanogaster by integrative inference of transcriptional networks. Genome Res 2012;22:1334-1349.

14 Shannon P, Markiel A, Ozier O, Baliga NS, Wang JT, Ramage D, Amin N, Schwikowski B, Ideker T: Cytoscape: A software environment for integrated models of biomolecular interaction networks. Genome Res 2003;13:2498-2504.

15 Huang da W, Sherman BT, Lempicki RA: Systematic and integrative analysis of large gene lists using david bioinformatics resources. Nat Protoc 2009;4:44-57.

16 Jeon S, Park YJ, Kwon YH: Genistein alleviates the development of nonalcoholic steatohepatitis in apoe(-/-) mice fed a high-fat diet. Molecular nutrition \& food research 2014;58:830-841. 


\section{Cellular Physiology Cell Physiol Biochem 2017;41:239-251 \begin{tabular}{ll|l} 
DOI: 10.1159/000456061 2017 The Author(s). Published by S. Karger AG, Basel & $\begin{array}{l}\text { ( 2017 } \\
\text { www.karger.com/cpb }\end{array}$
\end{tabular}}

Lou et al.: Transcriptional Co-Regulatory Network of NAFLD

-17 Brunt EM, Kleiner DE, Wilson LA, Belt P, Neuschwander-Tetri BA, Network NCR: Nonalcoholic fatty liver disease (nafld) activity score and the histopathologic diagnosis in nafld: Distinct clinicopathologic meanings. Hepatology 2011;53:810-820.

-18 Morello D, Lavenu A, Babinet C: Differential regulation and expression of jun, c-fos and c-myc protooncogenes during mouse liver regeneration and after inhibition of protein synthesis. Oncogene 1990;5:1511-1519.

19 Gokey NG, Lopez-Anido C, Gillian-Daniel AL, Svaren J: Early growth response 1 (egr1) regulates cholesterol biosynthetic gene expression. J Biol Chem 2011;286:29501-29510.

20 Smalling RL, Delker DA, Zhang Y, Nieto N, McGuiness MS, Liu S, Friedman SL, Hagedorn CH, Wang L: Genome-wide transcriptome analysis identifies novel gene signatures implicated in human chronic liver disease. Am J Physiol Gastrointest Liver Physiol 2013;305:G364-374.

21 Zhang Y, Hagedorn CH, Wang L: Role of nuclear receptor shp in metabolism and cancer. Biochim Biophys Acta 2011;1812:893-908.

-22 Zhang Y, Bonzo JA, Gonzalez FJ, Wang L: Diurnal regulation of the early growth response 1 (egr-1) protein expression by hepatocyte nuclear factor 4alpha (hnf4alpha) and small heterodimer partner (shp) crosstalk in liver fibrosis. J Biol Chem 2011;286:29635-29643.

-23 Huang J, Tabbi-Anneni I, Gunda V, Wang L: Transcription factor nrf2 regulates shp and lipogenic gene expression in hepatic lipid metabolism. Am J Physiol Gastrointest Liver Physiol 2010;299:G1211-1221.

24 Zhang Y, Andrews GK, Wang L: Zinc-induced dnmt1 expression involves antagonism between mtf-1 and nuclear receptor shp. Nucleic Acids Res 2012;40:4850-4860.

-25 Jung DY, Chalasani U, Pan N, Friedline RH, Prosdocimo DA, Nam M, Azuma Y, Maganti R, Yu K, Velagapudi A, O'Sullivan-Murphy B, Sartoretto JL, Jain MK, Cooper MP, Urano F, Kim JK, Gray S: Klf15 is a molecular link between endoplasmic reticulum stress and insulin resistance. PLoS One 2013;8:e77851.

-26 Takashima M, Ogawa W, Hayashi K, Inoue H, Kinoshita S, Okamoto Y, Sakaue H, Wataoka Y, Emi A, Senga Y, Matsuki Y, Watanabe E, Hiramatsu R, Kasuga M: Role of klf15 in regulation of hepatic gluconeogenesis and metformin action. Diabetes 2010;59:1608-1615.

-27 Han S, Zhang R, Jain R, Shi H, Zhang L, Zhou G, Sangwung P, Tugal D, Atkins GB, Prosdocimo DA, Lu Y, Han X, Tso P, Liao X, Epstein JA, Jain MK: Circadian control of bile acid synthesis by a klf15-fgf15 axis. Nat Commun 2015;6:7231.

-28 Veum VL, Dankel SN, Gjerde J, Nielsen HJ, Solsvik MH, Haugen C, Christensen BJ, Hoang T, Fadnes DJ, Busch C, Vage V, Sagen JV, Mellgren G: The nuclear receptors nur77, nurr1 and nor1 in obesity and during fat loss. Int J Obes (Lond) 2012;36:1195-1202.

29 Chen P, Li J, Huo Y, Lu J, Wan L, Li B, Gan R, Guo C: Orphan nuclear receptor nr4a2 inhibits hepatic stellate cell proliferation through mapk pathway in liver fibrosis. PeerJ 2015;3:e1518.

30 Wang Y, Viscarra J, Kim SJ, Sul HS: Transcriptional regulation of hepatic lipogenesis. Nat Rev Mol Cell Biol 2015;16:678-689.

-31 Majdalawieh A, Zhang L, Fuki IV, Rader DJ, Ro HS: Adipocyte enhancer-binding protein 1 is a potential novel atherogenic factor involved in macrophage cholesterol homeostasis and inflammation. Proc Natl Acad Sci U S A 2006;103:2346-2351.

-32 Li T, Diner BA, Chen J, Cristea IM: Acetylation modulates cellular distribution and DNA sensing ability of interferon-inducible protein ifi16. Proc Natl Acad Sci U S A 2012;109:10558-10563.

-33 Wang L, Zhang N, Wang Z, Ai DM, Cao ZY, Pan HP: Decreased mir-155 level in the peripheral blood of nonalcoholic fatty liver disease patients may serve as a biomarker and may influence lxr activity. Cell Physiol Biochem 2016;39:2239-2248.

-34 Font-Burgada J, Sun B, Karin M: Obesity and cancer: The oil that feeds the flame. Cell Metab 2016;23:48-62.

- 35 Mao Y, Cheng J, Yu F, Li H, Guo C, Fan X: Ghrelin attenuated lipotoxicity via autophagy induction and nuclear factor-kappab inhibition. Cell Physiol Biochem 2015;37:563-576.

-36 Hetherington AM, Sawyez CG, Zilberman E, Stoianov AM, Robson DL, Borradaile NM: Differential lipotoxic effects of palmitate and oleate in activated human hepatic stellate cells and epithelial hepatoma cells. Cell Physiol Biochem 2016;39:1648-1662.

-37 Consortium EP: An integrated encyclopedia of DNA elements in the human genome. Nature 2012;489:5774.

-38 Trierweiler C, Hockenjos B, Zatloukal K, Thimme R, Blum HE, Wagner EF, Hasselblatt P: The transcription factor c-jun/ap-1 promotes hbv-related liver tumorigenesis in mice. Cell Death Differ 2016;23:576-582.

-39 Schrem H, Klempnauer J, Borlak J: Liver-enriched transcription factors in liver function and development. Part ii: The c/ebps and d site-binding protein in cell cycle control, carcinogenesis, circadian gene 


\section{Cellular Physiology Cell Physiol Biochem 2017;41:239-251

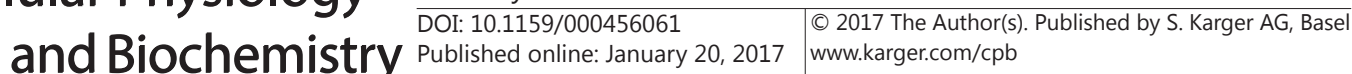

Lou et al.: Transcriptional Co-Regulatory Network of NAFLD

regulation, liver regeneration, apoptosis, and liver-specific gene regulation. Pharmacol Rev 2004;56:291330.

40 Jiao H, Zhu Y, Lu S, Zheng Y, Chen H: An integrated approach for the identification of hnf4alpha-centered transcriptional regulatory networks during early liver regeneration. Cell Physiol Biochem 2015;36:23172326.

41 Wang T, Chen T, Liang HY, Yan HT, Lin N, Liu LY, Luo H, Huang Z, Li NL, Liu WH, Tang LJ: Notch inhibition promotes fetal liver stem/progenitor cells differentiation into hepatocytes via the inhibition of hnf-1beta. Cell Tissue Res 2014;357:173-184.

$>42$ Wang T, You N, Tao K, Wang X, Zhao G, Xia N, Li N, Tang L, Liu W, Dou K: Notch is the key factor in the process of fetal liver stem/progenitor cells differentiation into hepatocytes. Dev Growth Differ 2012;54:605-617.

43 Chen YX, Weng ZH, Zhang SL: Notch3 regulates the activation of hepatic stellate cells. World J Gastroenterol 2012;18:1397-1403.

-44 Du B, Cawthorn WP, Su A, Doucette CR, Yao Y, Hemati N, Kampert S, McCoin C, Broome DT, Rosen CJ, Yang G, MacDougald OA: The transcription factor paired-related homeobox 1 (prrx1) inhibits adipogenesis by activating transforming growth factor-beta (tgfbeta) signaling. J Biol Chem 2013;288:3036-3047.

45 Kim YD, Kim SG, Hwang SL, Choi HS, Bae JH, Song DK, Im SS: B-cell translocation gene 2 regulates hepatic glucose homeostasis via induction of orphan nuclear receptor nur77 in diabetic mouse model. Diabetes 2014;63:1870-1880.

46 Schweitzer BL, Huang KJ, Kamath MB, Emelyanov AV, Birshtein BK, DeKoter RP: Spi-c has opposing effects to pu.1 on gene expression in progenitor b cells. J Immunol 2006;177:2195-2207.

-47 Kohyama M, Ise W, Edelson BT, Wilker PR, Hildner K, Mejia C, Frazier WA, Murphy TL, Murphy KM: Role for spi-c in the development of red pulp macrophages and splenic iron homeostasis. Nature 2009;457:318321.

-48 Kuraoka I, Suzuki K, Ito S, Hayashida M, Kwei JS, Ikegami T, Handa H, Nakabeppu Y, Tanaka K: Rna polymerase ii bypasses 8-oxoguanine in the presence of transcription elongation factor tfiis. DNA Repair (Amst) 2007;6:841-851.

49 Mackay LK, Minnich M, Kragten NA, Liao Y, Nota B, Seillet C, Zaid A, Man K, Preston S, Freestone D, Braun A, Wynne-Jones E, Behr FM, Stark R, Pellicci DG, Godfrey DI, Belz GT, Pellegrini M, Gebhardt T, Busslinger M, Shi W, Carbone FR, van Lier RA, Kallies A, van Gisbergen KP: Hobit and blimp1 instruct a universal transcriptional program of tissue residency in lymphocytes. Science 2016;352:459-463.

-50 Zhang C, Wang G, Zheng Z, Maddipati KR, Zhang X, Dyson G, Williams P, Duncan SA, Kaufman RJ, Zhang K: Endoplasmic reticulum-tethered transcription factor camp responsive element-binding protein, hepatocyte specific, regulates hepatic lipogenesis, fatty acid oxidation, and lipolysis upon metabolic stress in mice. Hepatology 2012;55:1070-1082.

-51 Sookoian S, Rosselli MS, Gemma C, Burgueno AL, Fernandez Gianotti T, Castano GO, Pirola CJ: Epigenetic regulation of insulin resistance in nonalcoholic fatty liver disease: Impact of liver methylation of the peroxisome proliferator-activated receptor gamma coactivator 1alpha promoter. Hepatology 2010;52:1992-2000.

52 Palumbo-Zerr K, Zerr P, Distler A, Fliehr J, Mancuso R, Huang J, Mielenz D, Tomcik M, Furnrohr BG, Scholtysek C, Dees C, Beyer C, Kronke G, Metzger D, Distler O, Schett G, Distler JH: Orphan nuclear receptor nr4a1 regulates transforming growth factor-beta signaling and fibrosis. Nat Med 2015;21:150-158.

53 Ye D, Li FY, Lam KS, Li H, Jia W, Wang Y, Man K, Lo CM, Li X, Xu A: Toll-like receptor-4 mediates obesityinduced non-alcoholic steatohepatitis through activation of $x$-box binding protein- 1 in mice. Gut 2012;61:1058-1067.

54 Ekstedt M, Hagstrom H, Nasr P, Fredrikson M, Stal P, Kechagias S, Hultcrantz R: Fibrosis stage is the strongest predictor for disease-specific mortality in nafld after up to 33 years of follow-up. Hepatology 2015;61:1547-1554.

55 Ye H, Liu W: Transcriptional networks implicated in human nonalcoholic fatty liver disease. Mol Genet Genomics 2015;290:1793-1804.

-56 Hyotylainen T, Jerby L, Petaja EM, Mattila I, Jantti S, Auvinen P, Gastaldelli A, Yki-Jarvinen H, Ruppin E, Oresic M: Genome-scale study reveals reduced metabolic adaptability in patients with non-alcoholic fatty liver disease. Nat Commun 2016;7:8994.

57 Sa R, Zhang W, Ge J, Wei X, Zhou Y, Landzberg DR, Wang Z, Han X, Chen L, Yin H: Discovering a critical transition state from nonalcoholic hepatosteatosis to nonalcoholic steatohepatitis by lipidomics and dynamical network biomarkers. J Mol Cell Biol 2016;8:195-206. 